\title{
To the 90th Birthday of Igor Ekhilievich Dzyaloshinskii
}

\author{
DOI: $10.1134 / \mathrm{S} 1063776121300014$
}

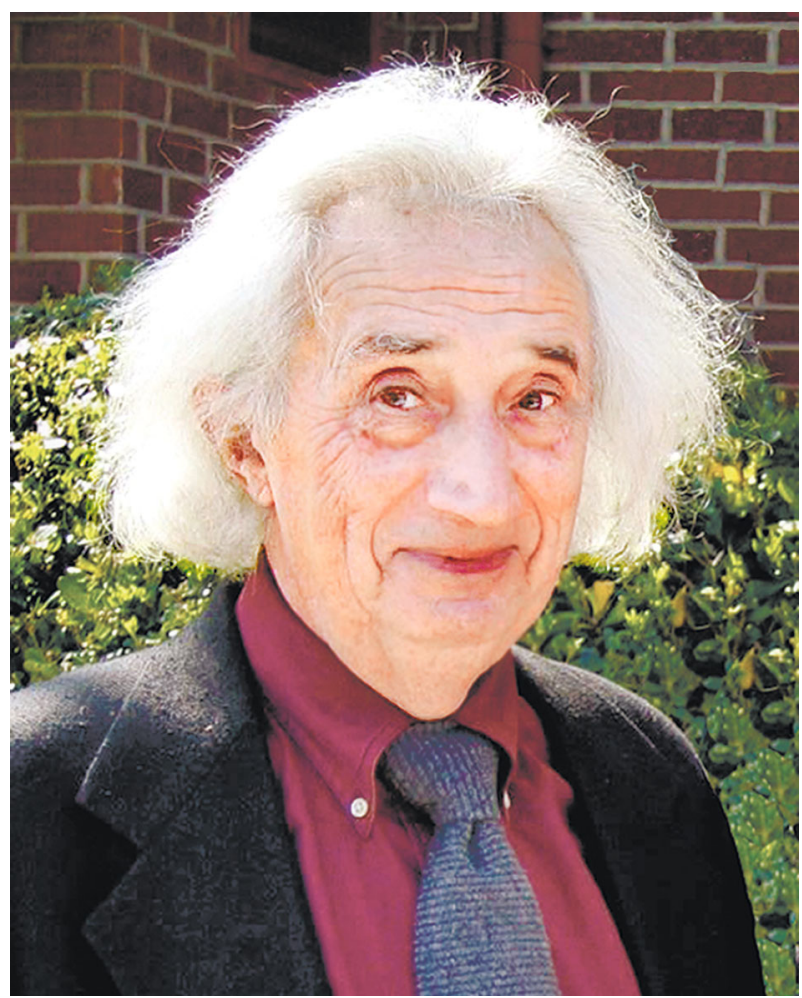

February 1, 2021 is the 90th birthday a prominent theoretical physicist, Igor Ekhilievich Dzyaloshinskii. At the start of his career, Dzyaloshinskii was a student and a closest associate of Lev Landau; now he is among the few active physicists who can call Landau his teacher. Speaking at the defense of Dzyaloshinskii's $\mathrm{PhD}$ thesis, Landau, who was hardly lavish in his praise of others, said, "The defender has already received so many commendations that yet another one is hardly necessary. But I have no reason to criticize him. I want to stress that in the course of recent years, Comrade Dzyaloshinskii has been one of the most talented young theoreticians whom I have met. He stands out for his creativity. It was his initiative to study the problems of ferromagnetism." Dzyaloshinskii authored numerous research papers, most of which were published in JETP; he served as Deputy Editor-in-chief of JETP for many years, together with E.M. Lifshitz. Early in his career, Dzyaloshinskii became a leader in the community of the physics of magnetic phenomena. Among his main results are the prediction of magnetoelectric effect, explanation of weak ferromagnetism in antiferromagnets (resulting from the interaction now known as Dzyaloshinskii-Moria interaction), the theory of helicoidal superstructures and commensurability effects. This last is also the subject of his recent papers: the advent of the age of "multiferroics" in the 2000s is actually based on the predictions made by a young scientist more than half a century ago.

Dzyaloshinskii's productivity, enthusiasm, and intellectual curiosity are remarkable for having covered apparently disparate subjects: the physics of magnetism, liquid crystals, polymers, exactly solvable models, and states with time-reversal symmetry violation. The contributions to this anniversary issue of JETP adequately reflect the scope of his research interests. It is worth noting that many of these works are actually based on the abovementioned predictions made by Dzyaloshinskii himself over the many years of his career in science. We are grateful to all the contributors to this issue and join them in wishing Dzyaloshinskii an excellent anniversary and good health. 